\title{
Coexistence of dark vector soliton Kerr combs in normal dispersion resonators
}

\author{
B. Kostet, ${ }^{1}$ Y. Soupart $\odot,{ }^{1}$ K. Panajotov $\odot,{ }^{2,3}$ and M. Tlidi ${ }^{1}$ \\ ${ }^{1}$ Département de Physique, Faculté des Sciences, Université Libre de Bruxelles (U.L.B.), CP 231, Campus Plaine, B-1050 Bruxelles, Belgium \\ ${ }^{2}$ Department of Applied Physics and Photonics (IR-TONA), Vrije Universiteit Brussels, Pleinlaan 2, 1050 Brussels, Belgium \\ ${ }^{3}$ Institute of Solid State Physics, 72 Tzarigradsko Chaussee Boulevard, 1784 Sofia, Bulgaria
}

(Received 28 July 2021; accepted 10 November 2021; published 29 November 2021)

\begin{abstract}
We investigate the formation of dark vector dissipative solitons in the presence of nonlinear polarization mode coupling in optical resonators subject to a coherent optical injection in the normal dispersion regime. This simple device is described by coupled Lugiato-Lefever equations. The stabilization of dark dissipative solitons is attributed to a front locking mechanism in the bistable regime as shown in a recent communication [B. Kostet et al., OSA Continuum 4, 1564 (2021)]. Here, we focus on a tristable homogeneous steady state regime. We show that two branches of dark dissipative solitons can coexist for a fixed value of the system parameters. These coexisting solutions possess different polarization states and different peak powers in the microresonator. We characterize their formation by drawing their bifurcation diagrams in regimes far from any modulational instability. It is shown that both branches of localized structures exhibit a heteroclinic collapsed snaking type of behavior. The coexistence of two vectorial branches of dark localized states is not possible without taking into account polarization degrees of freedom.
\end{abstract}

DOI: 10.1103/PhysRevA.104.053530

\section{INTRODUCTION}

The formation of localized structures often called dissipative solitons (DSs) is a patterning phenomenon that has been widely encountered in many far from equilibrium systems including fluid mechanics, optics, biology, and medicine [1]. These coherent and robust structures are often characterized by an intrinsic wavelength that is solely determined by the dynamical parameters and not by system size or boundary conditions. A classic example of nonequilibrium systems that undergo instabilities leading to the formation of DSs that are experimentally accessible is the field of nonlinear optics and laser physics [2,3].

In broad area optical resonators where light suffers diffraction, DSs consist of one or more spots of light in a two-dimensional transverse plane embedded in a homogeneous background [4]. However, in small area devices, by using a waveguide in both transverse directions, the intracavity field can be spatially stabilized. In this case, diffraction can be neglected in the modeling, and replaced by a natural chromatic dispersion of the Kerr medium which affects both the amplitude and the phase of light circulating within the cavity. The advantage of taking dispersion into account instead of diffraction is that chromatic dispersion varies with the optical wavelength and can be tuned. DSs in the temporal domain can thus be formed, and their optical spectra consist of equidistant lines forming frequency combs. The link between DSs and frequency comb generation has been established, reinforcing interest in the field of DS formation (see an excellent review by Lugiato et al. [5] in the theme issues [6,7]). Optical frequency combs generated by resonators possess a wide spectrum of applications in science and technology, ranging from high-precision spectroscopy to metrology and photonics [8]. Increasing interest has been paid to dissipative soliton frequency combs which correspond, in the time domain, to stable temporal localized structures or pulses that propagate within the resonator with the group velocity of light. They were reported experimentally in microresonators $[9,10]$.

When polarization degree of freedom is taken into account within Kerr resonators, new modulational instabilities can appear due to polarization degree of freedom [11-13], leading to domain wall vector solitons [14,15], symmetry breaking [16-21], and soliton bound states [22]. Recently, polarization properties of bright DS frequency combs have been the subject of investigation both theoretically [23-27] and experimentally [15]. Vector DSs are classified into two types: polarization-locked vector solitons and group-velocity-locked vector solitons. The former was first predicted [28] then experimentally verified $[29,30]$, and both have been investigated in mode-locked fiber lasers [31,32]. Vector DSs, however, do not consist solely of these two most common cases, but can also take the form of, e.g., polarization-precessing vector-locked solitons, as demonstrated in the spatial domain [33,34].

The paper is organized as follows. After an introduction, the well-known coupled Lugiato-Lefever equations (LLE [35]), describing a driven microcavity by taking into account polarization degrees of freedom, are introduced in Sec. II. We provide a detailed linear stability analysis of the homogeneous steady states by drawing a map as a function of the injection strength and the detuning parameter. In Sec. III, we discuss the mechanism of front locking leading to the formation of DSs in the normal dispersion regime, and we study the bifurcation structure in a tristable homogeneous steady state regime. The analysis of the bistable case has been reported 

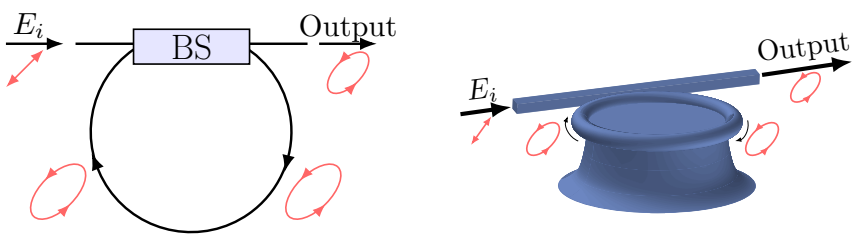

FIG. 1. Schematics of two typical optical resonators: (left) macroscopic fiber resonator and (right) microscopic toroidal resonator. Both devices are injected with linearly polarized light.

in [27]. In Sec. IV we analyze the situation where the two branches of dark localized structures exhibit an overlapping domain of stability. In this case, we identify a wide range of parameters in which we observe the coexistence of dark DSs with different polarization states and different peak powers. We conclude in Sec. V.

\section{COUPLED LUGIATO-LEFEVER MODEL AND ITS LINEAR STABILITY ANALYSIS}

We consider nonlinear optical resonators filled with a Kerr medium. Macro- and micro-resonators are schematically depicted in Fig. 1. A continuous wave with components along the transverse $x$ and $y$ axes, $E_{i_{x}}$ and $E_{i_{y}}$, is launched into the cavity through a beam splitter, propagates inside the Kerr medium, and experiences dispersion and the Kerr effect. At each round trip, the light inside the fiber is coherently superimposed with the input beam. This can be described by the propagation equations that consist of two coupled nonlinear Schrödinger equations and their boundary conditions. The formulation of this problem leads to infinite-dimensional maps that can be simplified to coupled LLE by applying the mean-field approximation for macroresonators such as all-fiber cavities [16] and for microresonators [12]. The dimensionless coupled LLE model reads

$$
\begin{aligned}
\partial_{t} E_{x, y}= & E_{i_{x}, i_{y}}+i\left(\left|E_{x, y}\right|^{2}+b\left|E_{y, x}\right|^{2}\right) E_{x, y} \\
& -\left(1+i \theta_{x, y} \mp \Delta \beta_{1} \partial_{\tau}-i \beta_{2} \partial_{\tau \tau}\right) E_{x, y}
\end{aligned}
$$

$E_{x, y}$ are the slowly varying envelopes for each polarization component of the intracavity field. We will focus on the case where the linearly polarized injected field has the same intensity for each polarization component, $E_{i_{x}}=E_{i_{y}}=E_{i} . b$ is the cross-phase modulation (XPM) coefficient between the two components. $\theta_{x}$ and $\theta_{y}$ are the frequency detunings for each polarization direction. $\Delta \beta_{1}$ corresponds to the group velocity mismatch, associated with the first-order dispersion, while the second-order dispersion coefficient $\beta_{2}$ is assumed to be the same in each direction. In the present study, we will focus on a macroscopic fiber resonator cavity. This allows us to set the cross-phase modulation coefficient as $b=2 / 3$ [36] and to reasonably approximate the group velocity mismatch $\Delta \beta_{1}$ as zero [37,38]. As we set $b<1$, the cavity operates in a regime where polarization domain walls do not occur $[14,15]$. We also consider the normal dispersion regime, $\beta_{2}=-1$. As we work here with dimensionless quantities, the axes of all figures are devoid of units.

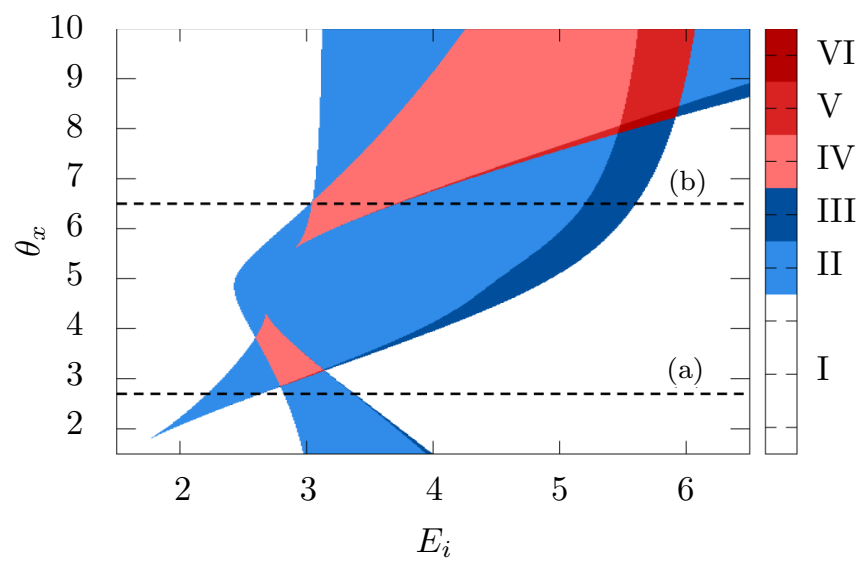

FIG. 2. Stability regions in the parameter space $E_{i}-\theta_{x}$. Parameters are $\theta_{y}=5$ and $\beta_{2}=-1$. Region I corresponds to monostability, with the presence of only one stable state. Region II corresponds to bistability between two stable states. Region III corresponds to bistability between one stable state and one modulationally unstable state. Region IV corresponds to tristability between three stable states. Region V corresponds to tristability between two stable states and one modulationally unstable state. Finally, region VI corresponds to tristability between one stable state and two modulationally unstable states. Examples of two consecutive bistable curves and of a tristable curve along the dashed lines (a) and (b) are shown in Figs. 3(a) and 3 (b) respectively.

The steady state continuous wave solutions of the coupled LLE (1) satisfy

$$
I_{i_{x}, i_{y}}=\left[1+\left(\theta_{x, y}-I_{x, y}-b I_{y, x}\right)^{2}\right] I_{x, y},
$$

where $I_{i_{x}, i_{y}}=E_{i_{x}, i_{y}}^{2}$ and $I_{x, y}=\left|E_{x, y}\right|^{2}$ are the intensities of injected fields and intensities of the intracavity fields, respectively. Equations (2) possess up to nine solutions for fixed values of the system parameters, five of which are physical. In a previous communication [27], we limited the analysis to parameter values for which the continuous wave $(\mathrm{CW})$ curve resembles the scalar case where the system develops the classical bistable $S$ curve. The linear stability analysis of the CW solutions with respect to a finite frequency of the form $\exp (i \omega \tau+\lambda t)$ is performed in the normal dispersion regime, i.e., $\beta_{2}>0$.

The results are summarized in the $E_{i}-\theta_{x}$ parameter space shown in Fig. 2. For small values of $\theta_{x}$, only simple bistability exists, as in the scalar case as mentioned above (region II). As the detuning $\theta_{x}$ is increased, two well-separated hysteresis loops, i.e., two separate regions of bistability, appear (light blue regions in Fig. 2). Increasing further $\theta_{x}$ causes the two distinct bistabilities to get closer until they overlap. In this case, a tristability region is generated, in light red on the map (region IV). Proceeding along with the map, this region collapses into the central bistability (II) zone. For even higher values of $\theta_{x}$, the tristability region (IV) reappears. Every region is bordered from below by a domain of coexistence with one or two modulationally unstable states (III, V, and VI). These domains with modulational instability (MI) sometimes get so thin that the spatial resolution of the plot does not allow us to see them. 

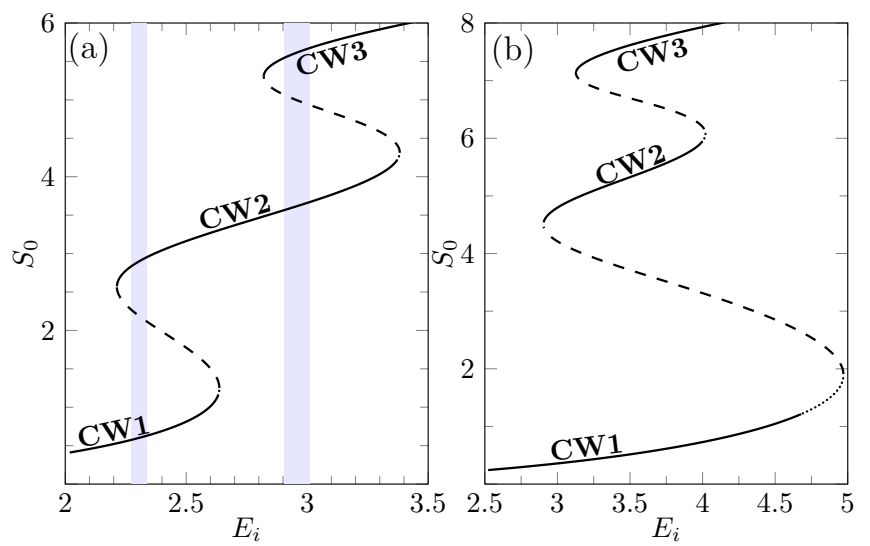

FIG. 3. Homogeneous steady states. Bistable curve (a) and tristable (b) obtained for $\theta_{y}=5$. These curves are taken along the dashed lines (a) and (b) in Fig. 2 corresponding to $\theta_{x}=2.7$ and $\theta_{x}=6.5$, respectively. Full lines correspond to stable states, dashed lines correspond to unstable states, and dotted lines correspond to modulationally unstable states. Highlighted regions in (a) correspond to zones where DSs can be stabilized.

In what follows, we consider two examples of bistable and tristable curves, Figs. 3(a) and 3(b), respectively. These figures are obtained from cuts along the dashed lines (a) and (b) in Fig. 2. The upper bistability collapses during the transition from region IV into region II in Fig. 2. This upper bistability reappears for higher values of the detuning parameter $\theta_{x}$. The small portions of MI can be seen as dotted curves in Figs. 3(a) and 3(b).

We first investigate the polarization properties of the homogeneous steady states shown in Fig. 3. The results are shown in Fig. 4 through the normalized Stokes parameters defined as $S_{0}=\left|E_{x}\right|^{2}+\left|E_{y}\right|^{2}, S_{1}=\left(\left|E_{x}\right|^{2}-\left|E_{y}\right|^{2}\right) / S_{0}, S_{2}=$ $2 \operatorname{Re}\left(E_{x} E_{y}^{*}\right) / S_{0}, S_{3}=-2 i\left(E_{x} E_{y}^{*}\right) / S_{0}$, where * stands for the complex conjugate. $S_{0}$ represents the total intensity of light in the resonators as seen above. $S_{1}$ corresponds to light polarized linearly along the axes $x$ and $y . S_{2}$ also corresponds to light

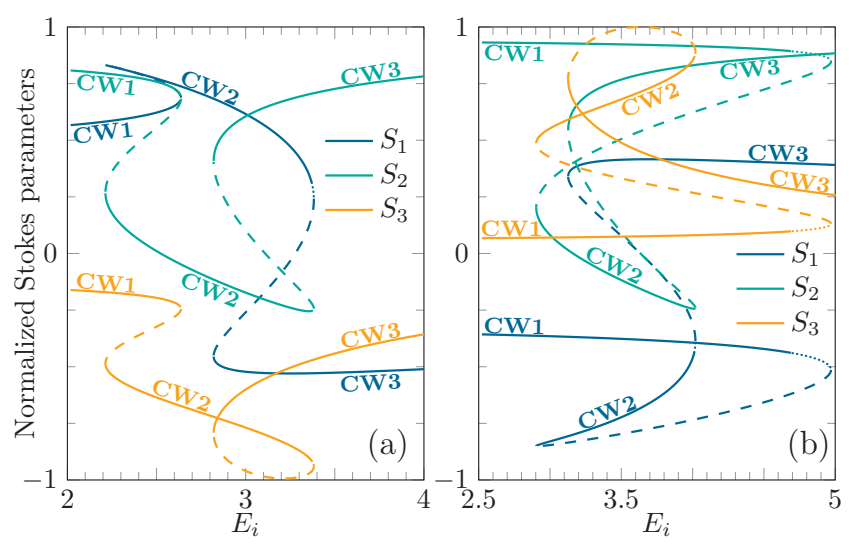

FIG. 4. Normalized Stokes parameters. (a) CW solutions plotted in Fig. 3(a). (b) CW solutions plotted in Fig. 3(b). The meaning of the dashed, dotted, and solid lines is the same as in Fig. 3. The parameters are the same as in Fig. 3. The injected light is linearly polarized, with $S_{1}=S_{3}=0$ and $S_{2}=1$. polarized linearly, but this time diagonally at $45^{\circ}$ with respect to the $x$ and $y$ axes. $S_{3}$ is indicative of the circularly polarized component of the light.

In Fig. 4(a), we start at low values of the injection with $S_{1}$ and $S_{2}$ significantly nonzero, while $S_{3}$ is close to zero, meaning that the light is mostly linearly polarized, with weak ellipticity. As the injection is increased, all three Stokes parameters undergo a hysteresis loop, leading to the first bistability between $\mathrm{CW}_{1}$ and $\mathrm{CW}_{2}$ as in Fig. 3. In particular, $S_{1}$ increases to almost one in a very narrow hysteresis loop, $S_{2}$ decreases to almost zero, and $S_{3}$ increases strongly in absolute value, so that $\mathrm{CW}_{2}$ gradually gets a strong ellipticity as the injection increases. Finally, a second hysteresis loop leads to $\mathrm{CW}_{3}$. For $S_{1}$ and $S_{2}$, the loops are large, ending on high values for both, with stronger $S_{2}$ than $S_{1}$. For $S_{3}$, this second bistability starts from a high value and appears with the curve folding on itself, before getting closer to zero, reaching almost the same values as in the beginning, so that $\mathrm{CW}_{3}$ will also be mostly linearly polarized, with ellipticity that decreases as the injection increases, but that stays higher than for $\mathrm{CW}_{1}$. In summary, both of the bistabilities from Fig. 4(a) occur between states that have significantly different polarization properties, especially regarding their ellipticity.

Following a similar process along the different curves in Fig. 4(b), we can see that in this case $\mathrm{CW}_{1}$ has an almost completely diagonal linear polarization with $S_{1}$ being low, $S_{2}$ being almost one and $S_{3}$ being almost zero. The polarization properties of $\mathrm{CW}_{1}$ barely change with the change in injection power. $\mathrm{CW}_{2}$ has a very strong ellipticity due to $S_{1}$ keeping high absolute values, $S_{2}$ being close to zero and $S_{3}$ being in the same range of values as $S_{1}$. Finally, $\mathrm{CW}_{3}$ has a constant $S_{1}$ at an intermediate value, while its $S_{2}$ increases to high values and $S_{3}$ gradually decreases to values close to zero as the injection power increases, leading to mostly diagonally linearly polarized light. In addition to the same conclusion as for the previous case, we can notice by comparing Figs. 4(a) and 4(b) that, without changing the injection intensity or the polarization of the injected light, merely changing the value of the detuning along one of the axis led to very significant changes in the polarization properties of the solutions, as well as in the size and shape of the hysteresis loops corresponding to the Stokes parameters.

\section{BIFURCATION STRUCTURE FOR THE DARK LOCALIZED STATES FOR DOUBLE BISTABILITY: COLLAPSED SNAKING}

In dispersive scalar Kerr micro- and macroresonators, temporal DSs can be generated in both anomalous and normal dispersion regimes. In the former case, DSs appear thanks to the subcritical nature of the modulation instability and their formation does not require a commutation process between the CW solutions. They can be generated in the monostable regime [4,39]. In the temporal regime, these DSs exhibit homoclinic snaking bifurcation diagrams that were first established close to zero dispersion wavelength [40]. In this case, dark DSs exhibit multistability behavior in a finite range of parameters referred to as the pinning region, where their bifurcation diagram consists of two snaking curves: one describes DSs with $2 n$ dips, while the other corresponds to $2 n+1$ dips 
(a)
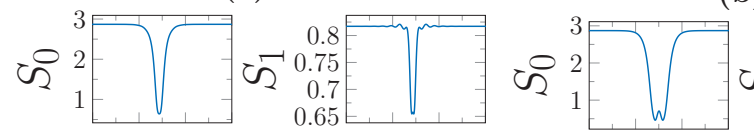

(b)
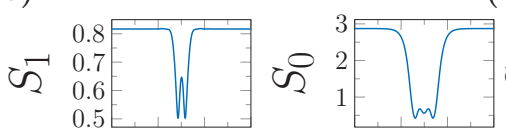

(c)
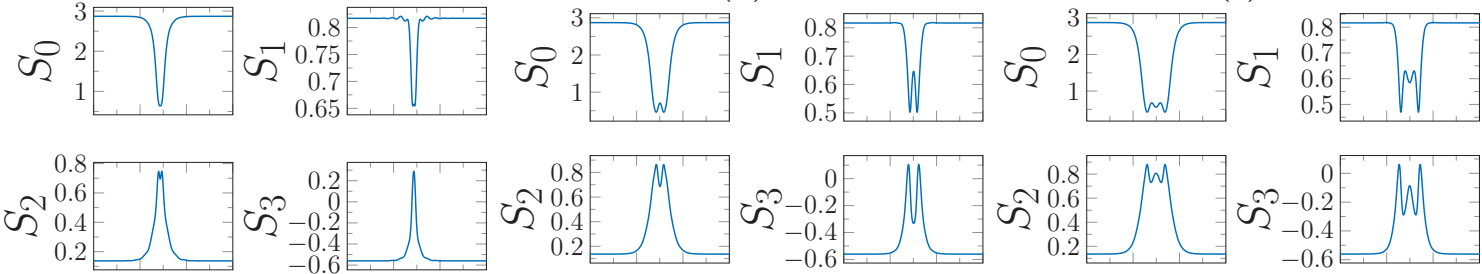

$\left(a^{\prime}\right)$
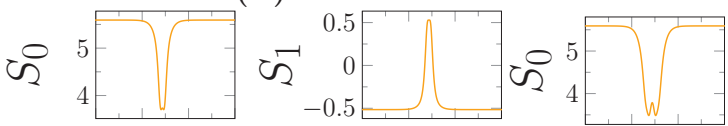

$\left(b^{\prime}\right)$
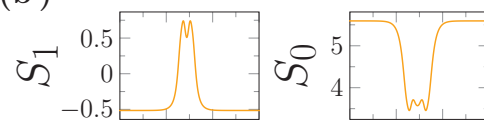

$\left(\mathrm{c}^{\prime}\right)$
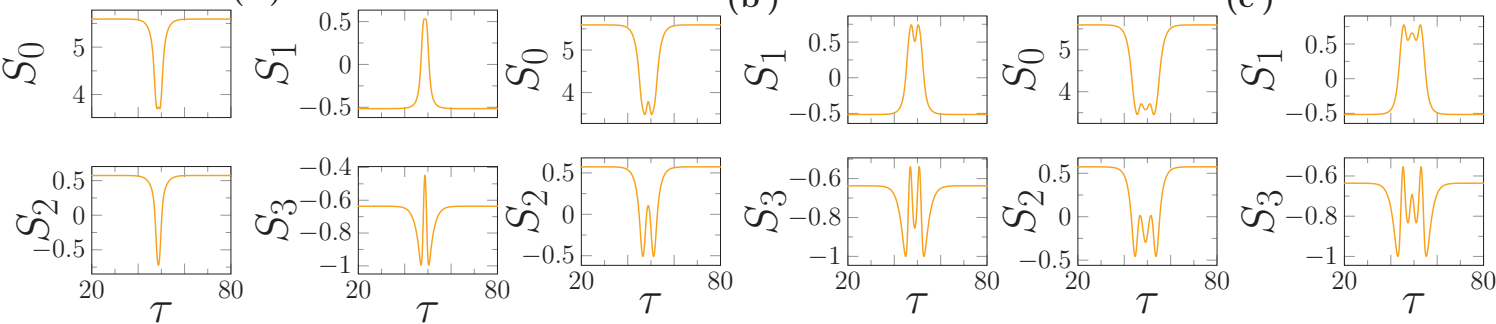

FIG. 5. Profiles of the Stokes parameters $S_{0}, S_{1}, S_{2}$, and $S_{3}$ as a function of the fast time $\tau$ for the stable DS solutions created in the highlighted regions of Fig. 3(a). The size of the system was taken as $L=100$, which corresponds to $83.6 \mu$ m in physical units. Profiles (a)-(c) correspond to the region highlighted on the left, while $\left(\mathrm{a}^{\prime}\right)-\left(\mathrm{c}^{\prime}\right)$ correspond to the region highlighted on the right. Injection amplitude values are $E_{i}=$ (a) 2.2917, (b) 2.2922, (c) 2.2926, ( a') 2.9394, ( b') 2.9406, ( c' 2.9406.

with $\mathrm{n}$ a positive integer. As one moves further along the snaking curve, the DS becomes better localized and acquires stability at the turning point where the slope becomes infinite. Afterward, the DS begins to grow along the fast temporal $\tau$ axis by adding extra dips symmetrically at either side. This growth is associated with back and forth oscillations across the pinning interval. This behavior is referred to as homoclinic snaking, and was established first in the spatial domain [41,42].

However, to generate dark DSs in the normal dispersion regime, the optical resonators must operate in the bistable regime. In this case the formation of dark DS results from a switching wave connecting the two stable $\mathrm{CW}$ solutions of the input-output characteristics curve [43-45]. These solutions are then of heteroclinic nature and their bifurcation diagram exhibits collapsed snaking [46,47]. More recently, this type of behavior has been reported in a vectorial driven all-fiber resonator [27] in a simple bistable regime. They appear when
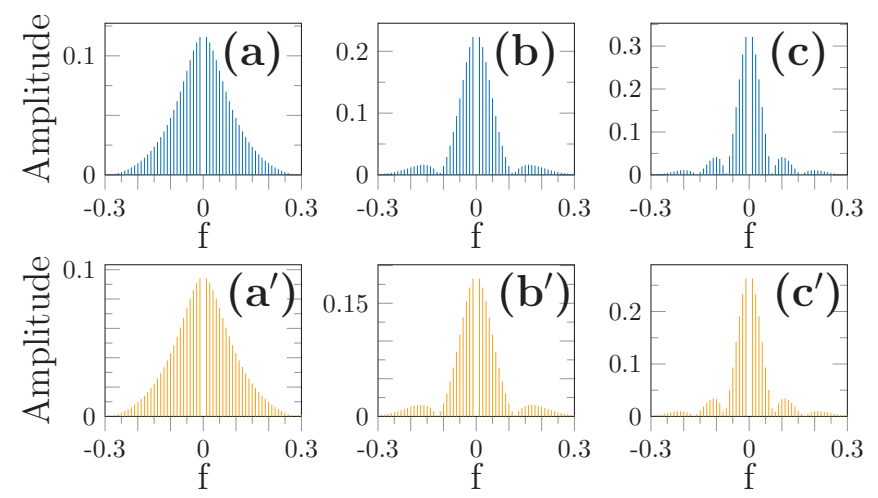

FIG. 6. Vector Kerr combs corresponding to Fourier transform of the stable DS solutions shown in Fig. 5. The free spectral range is equal to $2.45 \mathrm{THz}$. two fronts connecting stable CW states interact and lock into a stationary and robust temporal dark dissipative structure.

In what follows, we focus on heteroclinic solutions in a double bistability condition and in a regime far from the modulational instability, as shown in Fig. 3(a). We find stable heteroclinic DS solutions and we plot the profiles of their
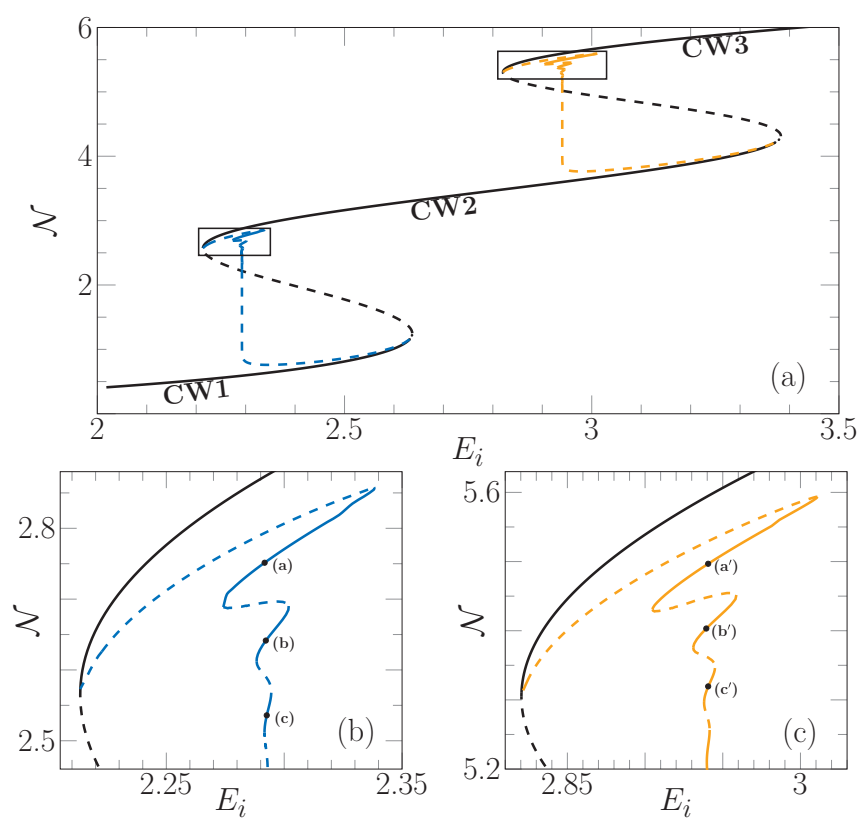

FIG. 7. Double heteroclinic snaking. (a) Bifurcation diagram showing the L2-norm $\mathcal{N}$ as a function of the injected field intensity $E_{i}$. Stable (unstable) states are denoted by solid (dashed) lines. MI states are denoted by dotted lines. Parameters are $\theta_{x}=2.7$ and $\theta_{y}=$ 5. (b) Close-up on the snaking curves collapsing onto the Maxwell point of the left bistability. (c) Close-up on the snaking curves collapsing onto the Maxwell point of the right bistability. 


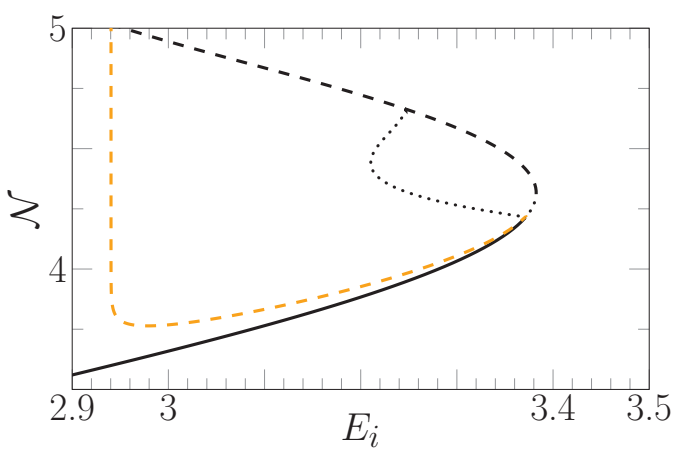

FIG. 8. Close-up showing the MI branch emerging from the end of the upper snaking curve from Fig. 7(a).

normalized Stokes parameters in Fig. 5. All these profiles correspond to heteroclinic connections between the homogeneous steady states shown in Figs. 3(a) and 4(a). For these DS solutions, the background corresponds to $\mathrm{CW}_{2}$ for the profiles of Fig. 5(a)-5(c) and to $\mathrm{CW}_{3}$ for the profiles of Fig. 5( $\left.\mathrm{a}^{\prime}\right)-5\left(\mathrm{c}^{\prime}\right)$. The connection for (a)-(c) is to $\mathrm{CW}_{1}$, and to $\mathrm{CW}_{2}$ for $\left(\mathrm{a}^{\prime}\right)-\left(\mathrm{c}^{\prime}\right)$. Note that the profiles of the $S_{3}$ component shown in Fig. 5 being nonzero is indicative of the presence of a circular component to the polarization in the resonator, so that the light is elliptically polarized. Furthermore, we can see that the different coexisting DS solutions connecting the same two CW states exhibit different polarization properties.

The spectral content of the total intensity profiles $S_{0}$ are optical frequency combs. The combs corresponding to the profiles from Fig. 5 are shown in Fig. 6. The comb lines are all equally spaced since the free spectral range, given by the inverse of the cavity round-trip time of their associated DSs is always the same. However, their envelopes depend on the exact profile of the considered solution. As the DS becomes wider when moving down the snaking curve, the comb becomes narrower. For the fundamental solution, the envelope of the spectrum follows an expected $\operatorname{sech}^{2}$ curve, but for each new solution appearing at each saddle-node (SN) bifurcation, the new bump emerging in the $S_{0}$ profile also brings a new bump in the envelope of the spectrum.

To construct the bifurcation diagram associated with vectorial DS, we fix the detuning parameters and chromatic dispersion coefficient, and let the injected field amplitude be the control parameter. We numerically simulate Eq. (1) to initiate a predictor-corrector continuation method in the parameter space [48]. Periodic boundary conditions are considered in all numerical simulations. The result is shown in the top panel of Fig. 7, where $\mathcal{N}=\int S_{0} / L d \tau$ is the normalized L2-norm of the electrical field with $L$ the size of the system. When increasing $E_{i}$, a branch of vectorial DS indicated in dark blue emerges from the upper limit point or turning point associated with the bistability curve that involves the $\mathrm{CW}_{1}$ and $\mathrm{CW}_{2}$ solutions. To visualize better this first family of vectorial DS, a close-up around the dark blue curve is shown in Fig. 7(b). This branch corresponds to the profiles (a)-(c) shown in Fig. 5. As we increase the injection intensity $E_{i}$, a region of monostability for $\mathrm{CW}_{2}$ arises, followed by another turning point leading to the second bistability between the $\mathrm{CW}_{2}$ and $\mathrm{CW}_{3}$ solutions, shown in light orange. A second distinct branch of vectorial DS emerges from the upper turning point of this new bistability, as shown in the zoom shown in Fig. 7(c). This branch corresponds to the profiles $\left(a^{\prime}\right)-\left(c^{\prime}\right)$ shown in Fig. 5. These two branches undergo a collapsed snaking: they emerge from the upper saddle-node bifurcation of their respective bistabilities, then oscillate with exponentially damped amplitude before collapsing on the Maxwell point where the fronts are stationary [49]. Finally, they end up connecting to the MI bifurcation point on the lower part of their respective bistabilities. This MI branch emerges from this point and connects to the unstable branch as shown in Fig 8. This figure indicates that the range of parameters for
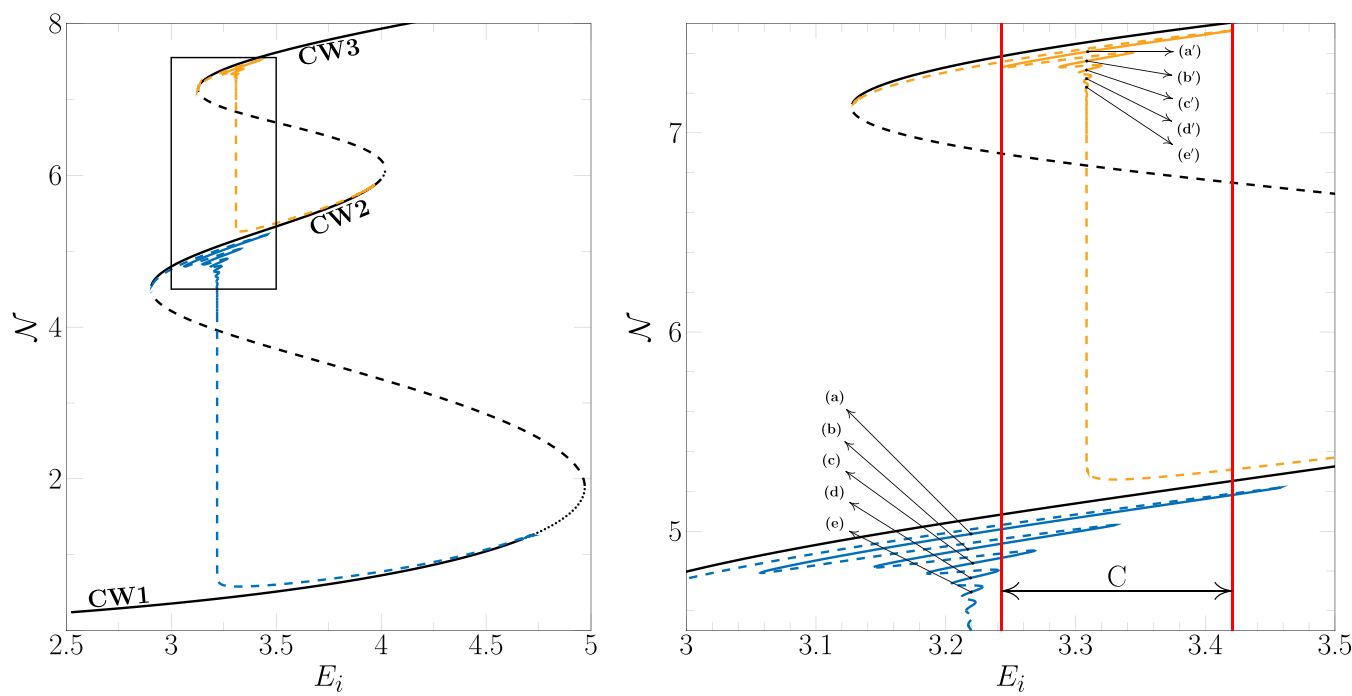

FIG. 9. Tristable heteroclinic snaking. Left panel: Bifurcation diagram showing the L2-norm $\mathcal{N}$ as a function of the injected field intensity $E_{i}$. Stable (unstable) states are denoted by solid (dashed) lines. MI states are denoted with dotted lines. Parameters are $\theta_{x}=6.5, \theta_{y}=4.5$. The size of the system was taken as $L=200$, which corresponds to $334.4 \mu \mathrm{m}$ in physical units. Right panel: Close-up on the snaking curves collapsing onto the Maxwell point of each respective bistability showing the coexistence region $C$. 
(a)
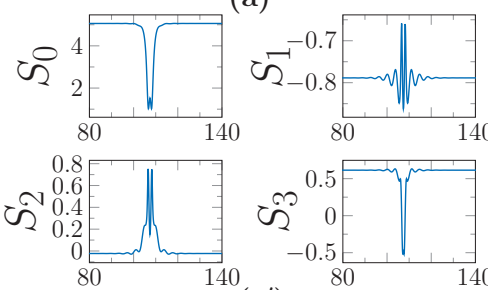

$\left(\mathbf{a}^{\prime}\right)$
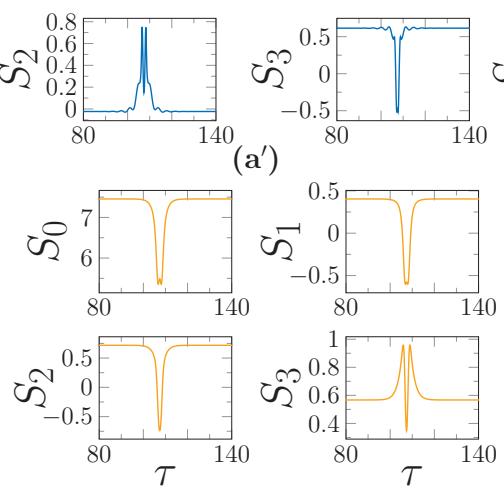

(b)
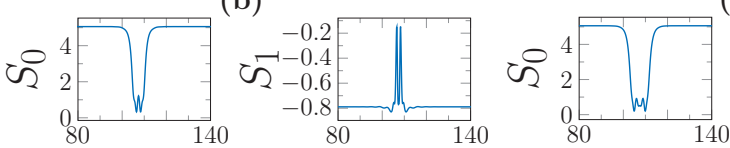

(c)
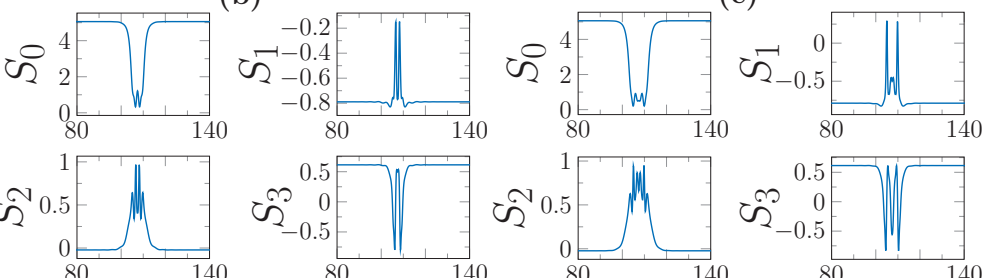

$\left(\mathrm{b}^{\prime}\right)$

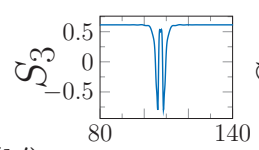

$\left(b^{\prime}\right)$
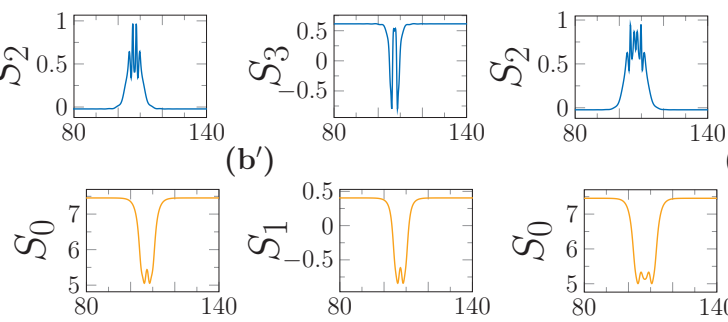

(c')

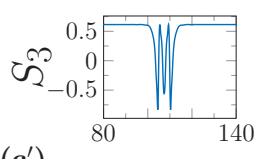

$\left(c^{\prime}\right)$
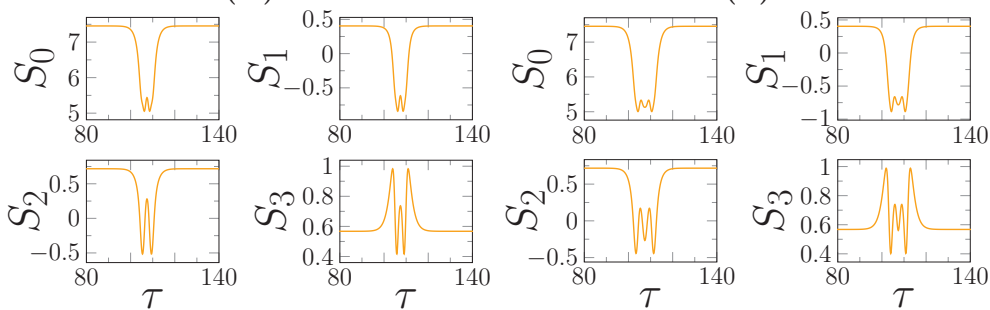

FIG. 10. Profiles of the Stokes parameters $S_{0}, S_{1}, S_{2}$, and $S_{3}$ as a function of the fast time $\tau$ for the stable solutions indicated in Fig. 9 . Parameters are the same as in Fig. 9. Injection amplitude values are $E_{i}=$ (a) 3.2195, (b) 3.217, (c) 3.2209, ( $\left.\mathrm{a}^{\prime}\right)$ 3.3094, ( b') 3.3087, ( $\mathrm{c}^{\prime}$ ) 3.3085 .

which our DSs exist is indeed far from the MI branch, so that they will not be affected by it.

This shows that, for this regime of parameters, two different branches, or families, of DS solutions coexist, but for different values of the injection field intensity so that the two families cannot coexist in the same physical system. These DS branches, during their oscillations, undergo several saddlenode (SN) bifurcations creating multiple different stable DSs. At each of these bifurcations, the new stable DS solution is characterized by the arising of a new bump at its bottom and becomes wider.

\section{COEXISTENCE BETWEEN TWO VECTORIAL BRANCHES OF DARK LOCALIZED STATES}

In the scalar case, i.e., without taking into account the polarization degree of freedom, the third-order dispersion allows for coexistence between bright and dark DSs [50]. This behavior occurs far from any MI, and the resulting DS connects the CWs of high and low intensities. However, both
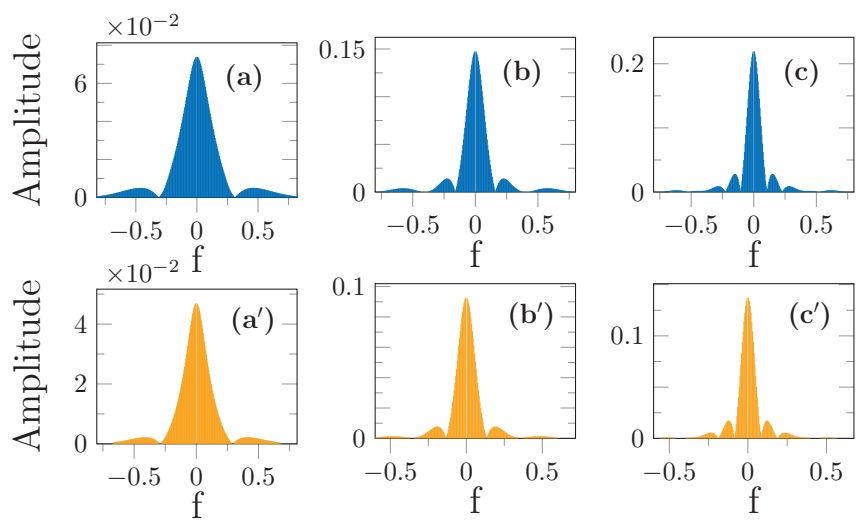

FIG. 11. Vector Kerr combs corresponding to the stable solutions shown in Fig. 10. The free spectral range is equal to $610 \mathrm{GHz}$. of these solutions move since the third-order dispersion breaks the reflection symmetry. Recently, it was shown that the LLE without high order dispersions support moving DSs [51,52]. The motion of DSs is attributed to the Raman delayed nonlocal response of the fiber. This effect can induce a coexistence between bright and dark DSs [53].

When considering the polarization degree of freedom, a coexistence between two types of bright localized structures has been reported in weakly birefringent all-fiber resonators subject to a linearly polarized optical injection, in the anomalous dispersion regime [23]. The resulting vector DSs do not only differ by their polarization states but also by their peak intensities. They are generated in a regime where two modulational instabilities with different frequencies appear in the system.

Recently, it was shown that, in the normal dispersion regime, the collapsed snaking structure of the DSs formed through switching waves interaction in the absence of MI allows for the coexistence between multiple types of dark localized structures, which also differ by their polarization states and by their shapes [27]. In that work, all coexisting DSs solutions connect the same upper and lower CW states.

In what follows, we consider the regime where the homogeneous steady states exhibit tristability. We constructed the bifurcation diagram associated with DSs in the same way as described above. The results are shown in Fig. 9. Once again, two branches of vectorial DSs emerge from the upper turning point of the two different bistability curves linking each pair of CW states, before going through damped oscillations and collapsing onto the MI point of the lower turning point. Figure 9 illustrates the remarkable property of domain $C$, namely that it is a region where the system exhibits a high degree of multistability. Besides the tristability associated with CW solutions, which are stable, two families of dark DSs can be obtained for fixed values of the system parameters. This behavior is caused by the two branches of heteroclinic collapsed snakings exhibiting an overlapping domain of stability, as indicated by region $C$. In this case, the two families are composed of the 


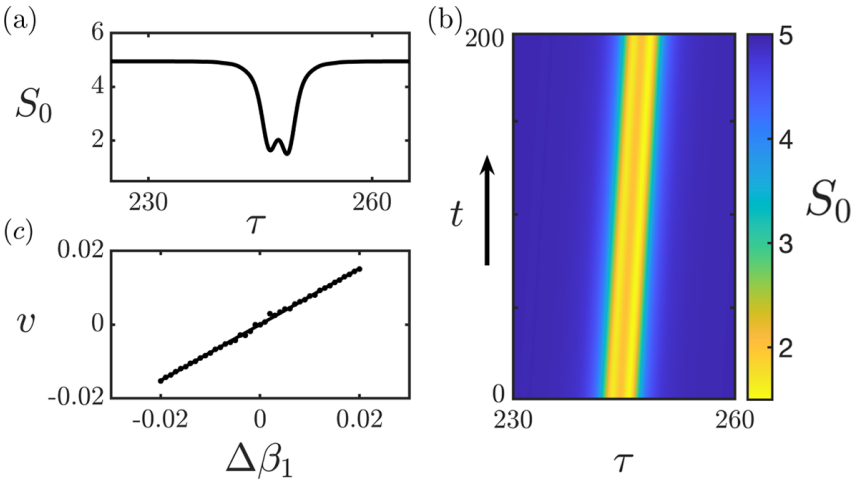

FIG. 12. Moving vector DS under the effect of group velocity mismatch. (a) Profile of an asymmetric DS due to nonzero group velocity mismatch. (b) Space-time map of the same DS showing the drifting motion. (c) Drift velocity $v$ as a function of the group velocity mismatch coefficient $\Delta \beta_{1}$.

solutions (a)-(c) from the bistability connecting $\mathrm{CW}_{1}$ and $\mathrm{CW}_{2}$ and the solutions $\left(\mathrm{a}^{\prime}\right)-\left(\mathrm{e}^{\prime}\right)$ from the bistability connecting $\mathrm{CW}_{2}$ and $\mathrm{CW}_{3}$, shown in the right part of Fig. 9. The profiles of the Stokes parameters corresponding to the coexisting DSs from the domain C are shown in Fig. 10. As in the previous case, these heteroclinic connections are between states with very different polarization properties, mostly regarding their ellipticity. The spectra of these solutions shown in Fig. 11 are optical frequency combs again, exhibiting the same properties as in the previous case.

In this study, we worked in the limit of zero group velocity mismatch between the field components. Depending on the birefringent characteristics of the medium, this effect can, however, become non-negligible. Introducing a nonzero group velocity mismatch in the coupled LLE (1) results in the symmetry $\tau \rightarrow-\tau$ being broken and leads to a drift motion of the DS along the cavity. This has been numerically studied from a reference frame moving at a speed that is the mean group velocity of the two components [see Eq. (1)]: $\Delta \beta_{1}=$ $\left(\beta_{1, x}-\beta_{1, y}\right) / 2$. Figures 12 (a) and 12(b) show the asymmetric profile and the drift over 1600 round trips, respectively. They have been generated for $\Delta \beta_{1}=0.02$, which corresponds to a physical value $\Delta \beta_{1}^{\prime} \approx 244 \mathrm{ps} / \mathrm{km}$ (or $3.3 \times 10^{-4}$ ps per round trip). Figure 12(c) presents the evolution of the drift velocity as the parameter $\Delta \beta_{1}$ is tuned. As expected, a linear dependency is observed and the direction of the drift (the sign of $v$ ) depends on the sign of the group velocity mismatch parameter. We can show that in our conditions and assuming a coupler transmission coefficient $\theta=10 \%$, internal losses of
$15 \%$, second-order dispersion coefficient $\beta_{2}=-20 \mathrm{ps}^{2} / \mathrm{km}$, and a refractive index $n=1.467$, if the dimensionless velocity mismatch amounts to 0.01 , the DS should perform 800000 cavity round trips before undergoing a drift corresponding to the cavity length. A study of this effect is ongoing and will be addressed in a future publication.

\section{CONCLUSIONS}

We have presented a bifurcation analysis of temporal dissipative solitons and their corresponding frequency combs, taking into account the polarization degree of freedom in resonators subjected to the continuous wave of linearly polarized injected fields. We have used the coupled Lugiato-Lefever equations to model this device that can either consist of a micro- or macroresonator. We have assumed that the optical resonator operates in a normal dispersion regime and is far from any modulational instability. We have presented a linear stability analysis of the model equations and their trivial homogenous steady state solutions.

Thanks to the front locking mechanism, we previously generated a single branch of DSs in a simple bistable regime that undergoes a collapsed snaking [27]. In this paper, we have focused the analysis on a double bistability curve with well-separated hysteresis loops and a tristable regime. In these regimes, two different branches of temporal dissipative solitons have been generated in the system. We have characterized vector DS solutions of Eq. (1) by drawing their bifurcation diagrams and by computing their Stokes parameters. Both branches of temporal dissipative solitons exhibit a collapsed snaking type of bifurcation. We have considered first the double bistability with well-separated hysteresis loops. However, when the system develops a tristability, the two collapsed snaking overlap. We have shown that in this case, the system develops a high degree of multistability of temporal dissipative solitons possessing different polarization states, different widths, and different peak powers in vectorial microresonators.

\section{ACKNOWLEDGMENTS}

K.P. acknowledges support by the Fonds Wetenschappelijk Onderzoek-Vlaanderen FWO (G0E5819N) and the Methusalem Foundation. M.T. acknowledges financial support from the Fonds de la Recherche Scientifique FNRS under Grant CDR No. 35333527 "Semiconductor optical comb generator." A part of this work is supported by the "Laboratoire Associé International" University of Lille-ULB on "Selforganisation of light and extreme events" (LAI-ALLURE).
[1] N. Akhmediev and A. Ankiewicz, Dissipative Solitons: From Optics to Biology and Medicine, Lecture Notes in Physics Vol. 751 (Springer, Berlin, 2008).

[2] Y. K. Chembo, D. Gomila, M. Tlidi, and C. R. Menyuk, Eur. Phys. J. D 71, 299 (2017).

[3] B. A. Malomed and D. Mihalache, Rom. J. Phys. 64, 106 (2019).
[4] A. J. Scroggie, W. J. Firth, G. S. McDonald, M. Tlidi, R. Lefever, and L. A. Lugiato, Chaos Solitons Fractals 4, 1323 (1994).

[5] L. Lugiato, F. Prati, M. Gorodetsky, and T. Kippenberg, Philos. Trans. R. Soc. A 376, 20180113 (2018).

[6] M. Tlidi, M. Clerc, and K. Panajotov, Philos. Trans. R. Soc. A 376, 20180114 (2018). 
[7] M. Tlidi, M. Clerc, and K. Panajotov, Philos. Trans. R. Soc. A 376, 20180276 (2018).

[8] T. Fortier and E. Baumann, Commun. Phys. 2, 153 (2019).

[9] P. Del'Haye, A. Schliesser, O. Arcizet, T. Wilken, R. Holzwarth, and T. J. Kippenberg, Nature (London) 450, 1214 (2007).

[10] T. Herr, V. Brasch, J. D. Jost, C. Y. Wang, N. M. Kondratiev, M. L. Gorodetsky, and T. J. Kippenberg, Nat. Photonics 8, 145 (2014).

[11] S. Wabnitz, Phys. Rev. A 38, 2018 (1988).

[12] T. Hansson, M. Bernard, and S. Wabnitz, J. Opt. Soc. Am. B 35, 835 (2018).

[13] J. Fatome, B. Kibler, F. Leo, A. Bendahmane, G.-L. Oppo, B. Garbin, S. G. Murdoch, M. Erkintalo, and S. Coen, Opt. Lett. 45, 5069 (2020).

[14] M. Haelterman and A. P. Sheppard, Opt. Lett. 19, 96 (1994).

[15] B. Garbin, J. Fatome, G.-L. Oppo, M. Erkintalo, S. G. Murdoch, and S. Coen, Phys. Rev. Lett. 126, 023904 (2021).

[16] M. Haelterman, S. Trillo, and S. Wabnitz, J. Opt. Soc. Am. B 11, 446 (1994).

[17] G. Millot, E. Seve, and S. Wabnitz, Phys. Rev. Lett. 79, 661 (1997).

[18] C. Cambournac, T. Sylvestre, H. Maillotte, B. Vanderlinden, P. Kockaert, P. Emplit, and M. Haelterman, Phys. Rev. Lett. 89, 083901 (2002).

[19] B. Garbin, J. Fatome, G.-L. Oppo, M. Erkintalo, S. G. Murdoch, and S. Coen, Phys. Rev. Research 2, 023244 (2020).

[20] L. Hill, G.-L. Oppo, M. T. M. Woodley, and P. Del'Haye, Phys. Rev. A 101, 013823 (2020).

[21] G. Xu, A. U. Nielsen, B. Garbin, L. Hill, G.-L. Oppo, J. Fatome, S. G. Murdoch, S. Coen, and M. Erkintalo, Nat. Commun. 12, 4023 (2021).

[22] P. Kockaert and M. Haelterman, J. Opt. Soc. Am. B 16, 732 (1999).

[23] E. Averlant, M. Tlidi, K. Panajotov, and L. Weicker, Opt. Lett. 42, 2750 (2017).

[24] F. Copie, M. T. M. Woodley, L. Del Bino, J. M. Silver, S. Zhang, and P. Del'Haye, Phys. Rev. Lett. 122, 013905 (2019).

[25] R. Suzuki, S. Fujii, A. Hori, and T. Tanabe, IEEE Photonics J. 11, 1 (2019).

[26] M. Saha, S. Roy, and S. K. Varshney, Phys. Rev. A 101, 033826 (2020).

[27] B. Kostet, S. S. Gopalakrishnan, E. Averlant, Y. Soupart, K. Panajotov, and M. Tlidi, OSA Continuum 4, 1564 (2021).

[28] N. Akhmediev, A. Buryak, and J. Soto-Crespo, Opt. Commun. 112, 278 (1994).
[29] S. T. Cundiff, B. C. Collings, N. N. Akhmediev, J. M. Soto-Crespo, K. Bergman, and W. H. Knox, Phys. Rev. Lett. 82, 3988 (1999).

[30] C. Mou, S. Sergeyev, A. Rozhin, and S. Turistyn, Opt. Lett. 36, 3831 (2011).

[31] S. Cundiff, B. Collings, and W. Knox, Opt. Express 1, 12 (1997).

[32] L. M. Zhao, D. Y. Tang, H. Zhang, X. Wu, and N. Xiang, Opt. Express 16, 9528 (2008).

[33] S. V. Sergeyev, Philos. Trans. R. Soc. A 372, 20140006 (2014).

[34] C. Mou, S. V. Sergeyev, A. G. Rozhin, and S. K. Turitsyn, Opt. Express 21, 26868 (2013).

[35] L. A. Lugiato and R. Lefever, Phys. Rev. Lett. 58, 2209 (1987).

[36] G. P. Agrawal, in Nonlinear Fiber Optics, edited by G. Agrawal, 5th ed. (Academic, Boston, 2013), pp. 193-244.

[37] D. Y. Tang, Y. F. Song, J. Guo, Y. J. Xiang, and D. Y. Shen, IEEE J. Sel. Top. Quantum Electron. 20, 42 (2014).

[38] A. U. Nielsen, B. Garbin, S. Coen, S. G. Murdoch, and M. Erkintalo, Phys. Rev. Lett. 123, 013902 (2019).

[39] M. Tlidi, P. Mandel, and R. Lefever, Phys. Rev. Lett. 73, 640 (1994).

[40] M. Tlidi and L. Gelens, Opt. Lett. 35, 306 (2010).

[41] A. R. Champneys, Physica D 112, 158 (1998).

[42] P. D. Woods and A. R. Champneys, Physica D 129, 147 (1999).

[43] S. Coen, M. Tlidi, P. Emplit, and M. Haelterman, Phys. Rev. Lett. 83, 2328 (1999).

[44] X. Xue, Y. Xuan, Y. Liu, P.-H. Wang, S. Chen, J. Wang, D. E. Leaird, M. Qi, and A. M. Weiner, Nat. Photonics 9, 594 (2015).

[45] B. Garbin, Y. Wang, S. G. Murdoch, G.-L. Oppo, S. Coen, and M. Erkintalo, Eur. Phys. J. D 71, 240 (2017).

[46] P. Parra-Rivas, E. Knobloch, D. Gomila, and L. Gelens, Phys. Rev. A 93, 063839 (2016).

[47] P. Parra-Rivas, D. Gomila, E. Knobloch, S. Coen, and L. Gelens, Opt. Lett. 41, 2402 (2016).

[48] A. Dhooge, W. Govaerts, and Y. A. Kuznetsov, ACM Trans. Math. Software 29, 141 (2003).

[49] P. Coullet, L. Gil, and D. Repaux, Phys. Rev. Lett. 62, 2957 (1989).

[50] P. Parra-Rivas, D. Gomila, and L. Gelens, Phys. Rev. A 95, 053863 (2017).

[51] M.G. Clerc, S. Coulibaly, and M. Tlidi, Phys. Rev. Research 2, 013024 (2020).

[52] M.G. Clerc, S. Coulibaly, P. Parra-Rivas, and M. Tlidi, Chaos 30, 083111 (2020).

[53] P. Parra-Rivas, S. Coulibaly, M. G. Clerc, and M. Tlidi, Phys. Rev. A 103, 013507 (2021). 